\title{
Novel melanocortin 4 receptor agonist causes weight loss in obese rhesus macaques
}

The melanocortin 4 receptor (MC4R) is known to be involved in the regulation of feeding and energy homeostasis. Previous attempts to target this pathway in the treatment of obesity have been hampered by the association of MC4R agonists with cardiovascular adverse events. A new study demonstrates that the novel MC4R

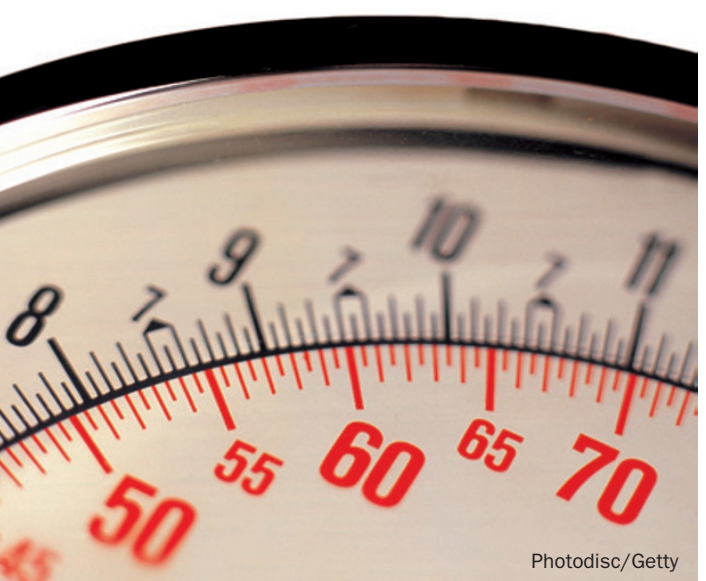

agonist RM-493 is safe and effective in rhesus macaques with diet-induced obesity. "These animals develop the full spectrum of metabolic diseases observed in humans, including central obesity, insulin resistance and hypertension, so they represent a powerful preclinical research model," explains corresponding author Kevin Grove (Oregon Health \& Science University, USA).

The study included 12 obese rhesus macaques that were maintained on a high-fat, high-calorie diet. The animals received RM-493 via a slow-release osmotic minipump for 8 weeks, followed by a 4-week washout period. The animals' weight, food intake and cardiovascular function were monitored.

The animals reduced their food intake for the first few weeks of treatment and although food intake then returned to pretreatment levels, the animals continued to lose weight throughout the treatment period (13.5\% body weight lost on average). Improvements in glucose sensitivity persisted into the washout period. Importantly, no increase in blood pressure or heart rate was observed. The animals also increased their physical activity levels and energy expenditure.

The authors note that RM-493 seems to have a mechanism of action that is distinct from other MC4R agonists. "The extent of the weight loss suggests that RM-493 shifts the metabolic setpoint in these obese primates, reducing appetite and increasing energy expenditure with good tolerability," concludes Grove. RM-493 is now progressing through phase I trials as a potential treatment of obesity.

\section{Claire Greenhill}

Original article Kievit, P. et al. Chronic treatment with
a melanocortin 4 receptor agonist causes weight loss,
reduces insulin resistance, and improves cardiovascular
function in diet-induced obese rhesus macaques. Diabetes
doi:10.2337/db12-0598

